\title{
Des lacunes en historiographie : La Vérendrye dans une perspective de relations homme-femme, de relations raciales et d'esclavage au début du Canada français, 1731-1749
}

\author{
Karlee A. Sapoznik-Evans
}

Volume 29, numéro 2, 2017

Territoire, langue et identité : présences nordiques dans l’Ouest canadien

URI : https://id.erudit.org/iderudit/1042269ar

DOI : https://doi.org/10.7202/1042269ar

Aller au sommaire du numéro

Éditeur(s)

Presses universitaires de Saint-Boniface (PUSB)

ISSN

0843-9559 (imprimé)

1916-7792 (numérique)

Découvrir la revue

Citer cet article

Sapoznik-Evans, K. A. (2017). Des lacunes en historiographie : La Vérendrye dans une perspective de relations homme-femme, de relations raciales et d'esclavage au début du Canada français, 1731-1749. Cahiers franco-canadiens de l'Ouest, 29(2), 457-487. https://doi.org/10.7202/1042269ar

\section{Résumé de l'article}

De nos jours, le nom de La Vérendrye figure sur de nombreux monuments commémoratifs, rues, parcs, écoles et bourses d'études prestigieuses tant canadiens qu'américains. Cependant, comme l'indique cet article, la documentation qui porte sur ses voyages et ses interactions turbulentes avec les peuples autochtones est incomplète, puisqu'elle est caractérisée par l'absence d'une analyse des échanges raciaux et de ceux entre hommes et femmes, et par une tradition de déni des faits et de création de mythes par rapport au commerce des esclaves chez les Canadiens français. L'examen soigneux de la participation de La Vérendrye au commerce des esclaves, et des façons que ses relations homme-femme et celles avec les peuples autochtones ont caractérisé sa vie entre 1731 et 1749, la période traitée par la présente étude, met en lumière le fonctionnement de la société coloniale française au Canada pendant la période allant du début du dix-huitième jusqu'au milieu de ce siècle. Comme souligné par cet article, les composantes non catholiques et non blanches faisaient partie intégrante de la société et de la culture coloniale du Canada français et exerçaient une influence sur elle. Les expériences de La Vérendrye démontrent que le Canada à cette époque laissait de la place à toutes sortes de complexités, de variantes et de contradictions dans les relations véritables entre hommes et femmes, et la Nouvelle-France était loin d'être une société égalitaire. L'esclavage y existait sous une forme institutionnelle tout comme l'esclavage existait au sud.
Tous droits réservés @ Centre d'études franco-canadiennes de l'Ouest (CEFCO) et Presses universitaires de Saint-Boniface (PUSB), 2017
Ce document est protégé par la loi sur le droit d'auteur. L’utilisation des services d'Érudit (y compris la reproduction) est assujettie à sa politique d'utilisation que vous pouvez consulter en ligne.

https://apropos.erudit.org/fr/usagers/politique-dutilisation/ 


\title{
Des lacunes en historiographie: La Vérendrye dans une perspective de relations homme-femme, de relations raciales et d'esclavage au début du Canada français, 1731-1749
}

\author{
par Karlee A. SAPOZNIK-EVANS*
}

\begin{abstract}
RÉSUMÉ
De nos jours, le nom de La Vérendrye figure sur de nombreux monuments commémoratifs, rues, parcs, écoles et bourses d'études prestigieuses tant canadiens qu'américains. Cependant, comme l'indique cet article, la documentation qui porte sur ses voyages et ses interactions turbulentes avec les peuples autochtones est incomplète, puisqu'elle est caractérisée par l'absence d'une analyse des échanges raciaux et de ceux entre hommes et femmes, et par une tradition de déni des faits et de création de mythes par rapport au commerce des esclaves chez les Canadiens français. L'examen soigneux de la participation de La Vérendrye au commerce des esclaves, et des façons que ses relations homme-femme et celles avec les peuples autochtones ont caractérisé sa vie entre 1731 et 1749 , la période traitée par la présente étude, met en lumière le fonctionnement de la société coloniale française au Canada pendant la période allant du début du dix-huitième jusqu'au milieu de ce siècle. Comme souligné par cet article, les composantes non
\end{abstract}

\footnotetext{
* $\mathrm{M}^{\text {me }}$ Sapoznik-Evans a obtenu son doctorat en histoire de l'Université York en Ontario. Elle a travaillé à la Faculté des arts de l'Université de Saint-Boniface comme chargée de cours de 2012-2014 et comme professeure adjointe de 2015-2016. Elle s'intéresse à la réconciliation, la justice sociale et l'esclavage sous toutes ses formes, notamment l'esclavage moderne. Native de Winnipeg, son intérêt pour La Vérendrye s'est éveillé en recevant la Bourse La Vérendrye pour l'excellence en histoire pendant ses études de premier cycle à l'ancien Collège universitaire de Saint-Boniface (maintenant USB) en 20052006.
} 
catholiques et non blanches faisaient partie intégrante de la société et de la culture coloniale du Canada français et exerçaient une influence sur elle. Les expériences de La Vérendrye démontrent que le Canada à cette époque laissait de la place à toutes sortes de complexités, de variantes et de contradictions dans les relations véritables entre hommes et femmes, et la Nouvelle-France était loin d'être une société égalitaire. L'esclavage y existait sous une forme institutionnelle tout comme l'esclavage existait au sud.

\begin{abstract}
Today, La Vérendrye's name is to be found on Canadian and American monuments, memorials, streets, parks, schools, and decorates prestigious scholarships. However, as this article reveals, the critical literature which focuses on his travels and turbulent interactions with Indigenous peoples is incomplete; it is marked by a lack of analysis of the intersection of gender with race, and by a tradition of denial and mythology surrounding the French-Canadian slave trade. Analysis of La Vérendrye's involvement in the slave trade, and the ways in which gender and Indigenous relations characterized his life in the period from 1731 to 1749 , the focus of the present study, sheds light on the functioning of early to mid-eighteenthcentury French colonial society in Canada. As this article emphasizes, non-Catholic, non-white elements formed an indispensable and influential part of French Canadian colonial society and culture. As evidenced by La Vérendrye's experiences, all sorts of complexity, diversity, and contradiction existed in the real-world relations of men and women, and New France was far from an egalitarian society. Slavery was institutionalized there just as it was to the south.
\end{abstract}

Les historiens modernes dépeignent souvent Pierre Gaultier de Varennes, et de La Vérendrye en tant qu'explorateur notable du XVIII siècle ayant joué un rôle de premier plan dans la «découverte» et la pénétration par les Français d'une grande partie de ce qui est maintenant $l^{\prime}$ Ouest canadien ${ }^{1}$. De nos jours, le nom de La Vérendrye figure sur de nombreux monuments commémoratifs, rues, parcs, écoles et bourses d'études prestigieuses tant canadiens qu'américains ${ }^{2}$. Il est 
particulièrement bien connu et honoré au Manitoba pour son arrivée au confluent des rivières Rouge et Assiniboine le 24 septembre 1738. En 1938, les villes de Winnipeg et de SaintBoniface ont organisé des célébrations du bicentenaire de ce passage, qui comprenaient un défilé et le dévoilement d'un monument en l'honneur de La Vérendrye. Cet évènement de neuf jours avait pour but, selon le programme souvenir, de «saluer les réalisations d'un des grands hommes de ce monde, l'Explorateur de l'Ouest» qui a «découvert et ouvert à la civilisation» la moitié ouest du continent nord-américain, «non pas par la force des armes, mais par la force de son caractère, par son commerce honnête, par le génie de son leadership, et par sa capacité de souder des amitiés et de les maintenir, par sa ténacité, sa détermination, sa patience sans égale, sa tolérance et sa force d'âme» [notre traduction] ${ }^{3}$. De maintes façons, les réalisations de La Vérendrye, et en particulier son courage et sa détermination à parcourir 2575 kilomètres par canoë de Montréal au lac Winnipeg, sont de faits remarquables.

Cependant, la documentation historiographique qui porte sur ses voyages et ses interactions turbulentes avec les peuples autochtones est incomplète. En effet, celle-ci se caractérise par l'absence d'une analyse des échanges raciaux et des échanges entre hommes et femmes, et par une tradition de déni des faits et de création de mythes par rapport au commerce des esclaves chez les Canadiens français. Un examen soigneux de la participation de La Vérendrye au commerce des esclaves, et des façons que ses relations homme-femme et celles avec les peuples autochtones ont caractérisé sa vie entre 1731 et 1749 , la période traitée par la présente étude, mettra en lumière le fonctionnement de la société coloniale française au Canada à partir du début du XVIII e siècle jusqu'au milieu de ce siècle. Traditionnellement, l'histoire du Régime français au Canada est l'histoire d'hommes blancs, une histoire où les femmes, les Autochtones et les noirs jouent des rôles de second plan ${ }^{4}$. À l'instar de certaines études publiées récemment, cet article cherchera à mieux inclure les autres groupes qui peuplaient la Nouvelle-France à l'époque ${ }^{5}$. Toutefois, il ne se penchera pas sur la sexualité, la violence ou l'environnement homosocial du commerce des fourrures en Nouvelle-France. Alors que ces sujets ont été examinés en profondeur ailleurs, on a porté moins d'attention à l'esclavage, à la notion de race et aux relations 
homme-femme en ce qui concerne La Vérendrye. Ce natif de Trois-Rivières avait servi dans l'armée française avant de devenir commerçant de fourrures, a pris une femme anishinabe, selon la tradition orale, et a fini par posséder au moins trois esclaves.

L'esclavage au Canada est longtemps resté un sujet négligé en histoire. Dans son ouvrage Histoire du Canada (1846), François Garneau a diffusé le mythe selon lequel l'esclavage n'a jamais existé en Nouvelle-France. Il a félicité le roi Louis XIV et le clergé colonial français d'avoir épargné le Canada français de cette «grande et terrible peste» ${ }^{6}$.

Suivant son exemple, d'autres ont soutenu qu'il n'y avait jamais eu d'esclavage en Nouvelle-France, et ce, malgré les preuves historiques: on a de fait dénombré au moins 4000 esclaves, dont les deux tiers étaient autochtones. L'idée fausse selon laquelle les premiers Africains dans la colonie sont venus en tant que réfugiés de l'esclavage du Sud persiste chez de nombreux Canadiens et ailleurs dans le monde ${ }^{7}$. Bien qu'il y ait un certain progrès, à cet égard, plusieurs s'imaginent que le Canada français, en raison d'une combinaison quelconque de climat, de population limitée ou de moralité chrétienne, a choisi de ne pas s'engager dans le commerce des esclaves. D'autres ouvrages se sont penchés sur les élites politiques de la NouvelleFrance, décrivant l'esclavage noir en termes d'enjeu secondaire dans la vie des Canadiens de peau blanche et tenant l'esclavage autochtone pour acquis, une sorte de conséquence inévitable de la colonisation et des guerres autochtones. Le présent article cherche à illustrer que l'idée de l'esclavage a été réprimée, décrite en termes voilés, et masquée par des étiquettes telles que «la découverte», «l'exploration» et «la colonisation».

Dans cette optique, un élément important à considérer en lisant les lettres de La Vérendrye et de ses contemporains est celui de la nature «construite» de la correspondance et de la rédaction de rapports. Carolyn Podruchny a suggéré que les analyses historiques doivent «lire au-delà des mots» et examiner les intentions manifestes d'hommes de la classe bourgeoise qui écrivent en ayant le pouvoir de diffuser de l'information ${ }^{8}$. Il faut regarder au-delà des partis-pris dans ces sources, car elles ont tendance à créer des suppositions par rapport à la hiérarchie sociale, à la relation entre les sexes et à la race. Une autre façon de lire au-delà des mots d'auteurs bourgeois masculins est de 


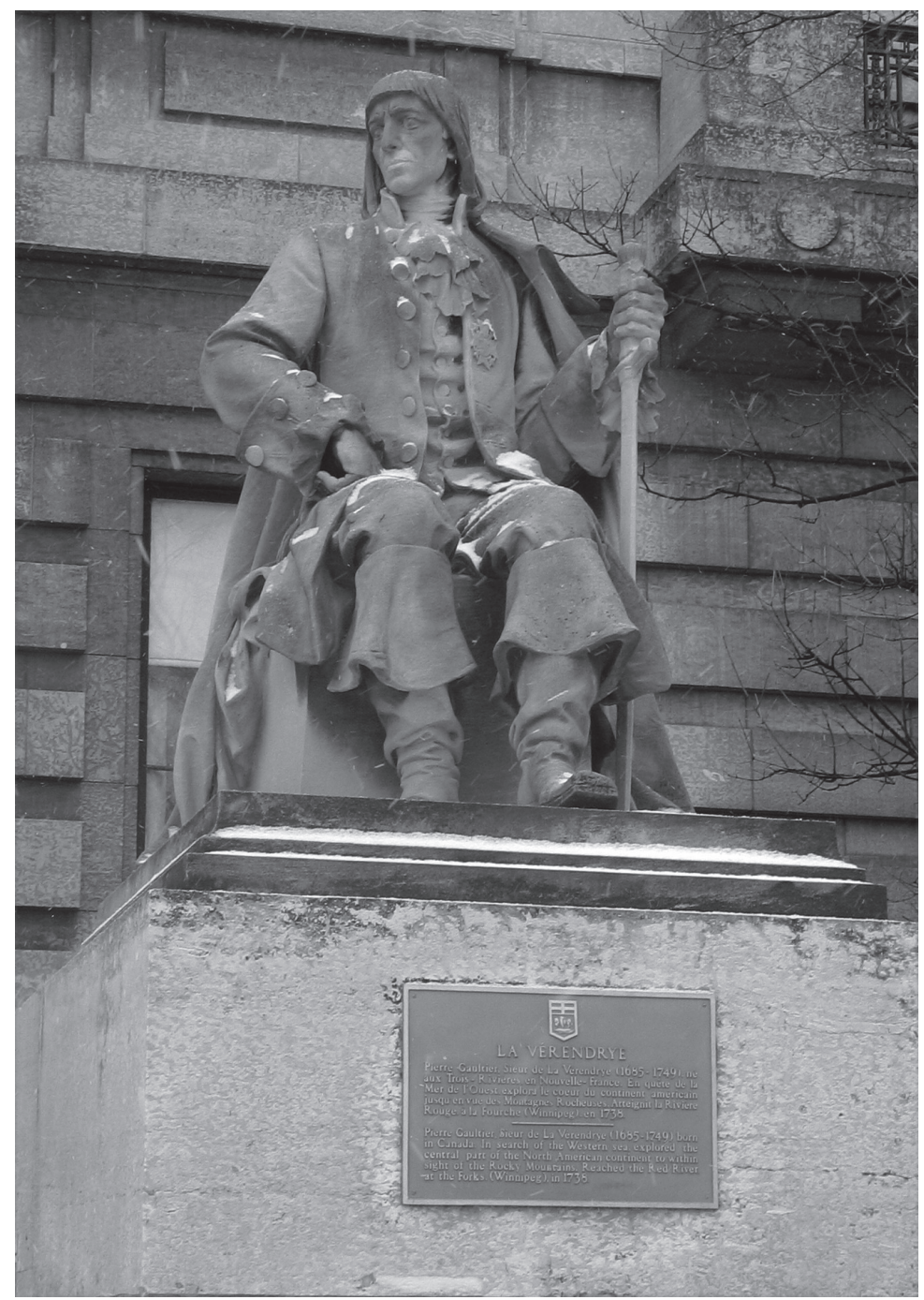

La Vérendrye protège l'entrée est de la législature provinciale à Winnipeg (Manitoba).

Photo: G. Goldsborough. 
décortiquer le sens des rituels. Le commerce des esclaves n'était pas simplement une coutume pittoresque et sentimentale. Dans les faits, l'échange rituel de cadeaux, y compris des esclaves, produisait et entretenait la solidarité communautaire en Nouvelle-France. Comme l'explique Podruchny, «les rituels peuvent créer, exprimer, enseigner et rappeler aux participants le sens et les valeurs de leur communauté et de leur identité [notre traduction] $»^{9}$. Dans le but de saisir l'étendue et la variété des relations qui existaient en Nouvelle-France dans les années 1730 et 1740, j'ai lancé mon filet le plus loin possible en termes de documentation secondaire, et je me suis concentrée sur les écrits de Carolyn Podruchny et Allan Greer. Dans mon analyse, j'essaie de lire à contre-courant dans les lettres et les rapports des autorités gouvernementales et des dignitaires ecclésiastiques qui font partie des documents primaires sur La Vérendrye recueillis par Lawrence Burpee et Antoine Champagne.

Depuis l'époque de Jacques Cartier (1491-1557), les uns après les autres, les explorateurs ont cherchéla route vers l'Orient, car on croyait que celle-ci raccourcirait énormément les voyages transatlantiques et contournerait les monopoles commerciaux de la Méditerranée, ce qui permettrait aux Français de récolter d'immenses bénéfices économiques. D,abord nébuleux, le concept de la mer de l'Ouest s'est précisé au début du XVIII ${ }^{e}$ siècle alors que les Français avaient une idée plus claire de la géographie de $1^{\prime}$ Amérique du Nord ${ }^{10}$.

Né à Trois-Rivières le 17 novembre 1685, La Vérendrye a combattu avec les Français dans des luttes armées entre la France et l'Angleterre en Nouvelle-Angleterre et à Terre-Neuve en 1704 et en 1705. À l'automne de 1707, il a pris le bateau pour la France afin de servir dans la guerre de Succession d'Espagne. Gravement blessé par épée et par balle pendant la bataille de Malplaquet, il a été laissé pour mort et est devenu prisonnier de guerre pendant quinze mois. Il est retourné en NouvelleFrance en 1711, où il a reçu le grade de lieutenant. En 1712, La Vérendrye a épousé sa fiancée, Marie-Anne Dandonneau du Sablé, et a repris le commerce desfourrures, se joignant dans une entreprise à son frère Jacques-René en 1726. Plus tard, La Vérendrye a accepté le poste de vice-commandant de la poste du Nord dans la région du lac Supérieur; il a fini par en devenir le commandant. C'est à cette époque qu'il a commencé à croire 
fermement que l'exploration du lac Winnipeg et de la «grande rivière de l'Ouest» mènerait à la «découverte» de l'océan Pacifique. En 1727, l'esclave d'un chef âgé du nom de Vieux Crapaud lui a décrit la terre des Mandans, un peuple censé savoir où était située la mer de l'Ouest ${ }^{11}$. Un an plus tard, Pierre Gaultier de Varennes, sieur de La Vérendrye a demandé au gouverneur Charles de Beauharnois la permission de s'aventurer vers l'Ouest. En 1731, il a été autorisé par la Cour royale de France à quitter Montréal en direction du lac Ouinipigon afin d'établir des postes de traite dans l'ouest du Canada. Il devait en même temps souder des alliances avec les Premières nations, organiser le commerce des fourrures de façon à ce qu'il soit concurrentiel avec le système lucratif des Anglais dans la région de la baie d'Hudson et «découvrir» une route menant à la mer de l'Ouest. Afin de financer ses expéditions, la Couronne lui octroya un monopole sur le commerce des fourrures à l'ouest du lac Supérieur. Les quatre fils de La Vérendrye, Jean-Baptiste, Pierre, François et Louis-Joseph, ainsi que son neveu, La Jemmeraye, prirent part à ses explorations au nord du quarantième parallèle et à louest des Grands Lacs et de la rivière Mississippi, jusqu'à sa mort en $1749^{12}$.

Jusqu'au milieu du XIX ${ }^{\mathrm{e}}$ siècle, la source principale d'information sur La Vérendrye était les Mémoires sur le Canada, depuis 1749 jusqu'à 1760 de Louis-Léonard Courville. La Vérendrye y est dépeint sous les traits d'un homme motivé par des intérêts égoïstes et qui, n'ayant ni éducation ni habiletés naturelles, est mal adapté à la carrière d'explorateur. Les bases d'une réévaluation de cette image ont été jetées par l'archiviste français Pierre Margry, qui a découvert une grande quantité de documents concernant La Vérendrye. En 1852, Margry publie un bref article révisionniste intitulé «Les Varennes de La Vérendrye» dans Le Moniteur Universel. À cause de cet article, et plus particulièrement des documents que Margry publie subséquemment, La Vérendrye devient une des figures de proue du Régime français ${ }^{13}$. Dans les années 1960, plusieurs auteurs publient des biographies de La Vérendrye. Il est représenté traditionnellement par ses deux principaux biographes, Antoine Champagne et N. M. Crouse, d'abord et avant tout comme un explorateur mal compris par le gouvernement français à Versailles. Crouse et Champagne rejettent la thèse selon laquelle La Vérendrye se préoccupait d'abord du commerce des 
fourrures, et ils refusent d'accepter le doute qui régnait chez les autorités françaises qu'il n'avait pas envie d'explorer l'Ouest canadien. Jean-Frédéric Phélyppeaux, comte de Maurepas, ministre de la Marine de France, remettait continuellement en question l'engagement de La Vérendrye envers «l'exploration» et l'accusait de considérer les profits avant ses devoirs à la Couronne française ${ }^{14}$. Le gouverneur de la Nouvelle-France, Charles, marquis de Beauharnois, est cependant demeuré un allié constant de La Vérendrye lors de ses batailles avec la Cour royale de France.

Le soutien que Beauharnois apportait à La Vérendrye est peut-être influencé par les profits qu'il tirait du commerce des fourrures et des esclaves, profits que Maurepas avait conseilléaux autorités de la Nouvelle-France de réduire.Selon Brett Rushforth, Beauharnois possédait au moins huit esclaves Renard ${ }^{15}$. En octobre 1735, Maurepas, furieux du manque de progrès réalisé par La Vérendrye, proclame que la mer de l'Ouest aurait été «découverte» depuis longtemps si les hommes de La Vérendrye portaient moins d'attention à la «mer du Castor ${ }^{16}$ ». Antoine Champagne blâme ce portrait négatif de La Vérendrye dressé par Maurepas sur les rapports qu'il recevait de commerçants en pelleterie jaloux ${ }^{17}$. Dans l'ensemble, Champagne propose que les autorités à Versailles n'étaient pas assez reconnaissantes envers La Vérendrye, ni d'avoir été responsable d'une hausse des profits du commerce des fourrures, ni de la possession par la France d'un territoire immense et riche, ni pour son rôle à ouvrir le chemin vers la mer de l'Ouest, ni pour avoir enlevé «des mains des barbares un bon nombre d'esclaves utiles à la colonie $^{18}{ }_{\gg}$. Offrant des preuves peu convaincantes, Champagne suggère qu'il existait au Canada français une forme d'esclavage plus bénigne qu'ailleurs; que les esclaves en Nouvelle-France vivaient relativement heureux; que presque tous aimaient leurs maitres, qui les aimaient bien en retour ${ }^{19}$.

Champagne reconnait que La Vérendrye était impliqué dans l'institution de l'esclavage, raportant par exemple que celui-ci avait un esclave à son service lorsqu'il dirigeait l'expédition Mandan en 1738, cependant il en fait peu de cas, et considère cette participation à la traite des esclaves comme une simple coutume de l'époque ${ }^{20}$. S'il est vrai qu'à bien des égards La Vérendrye n'était qu'un Français ordinaire participant à un 


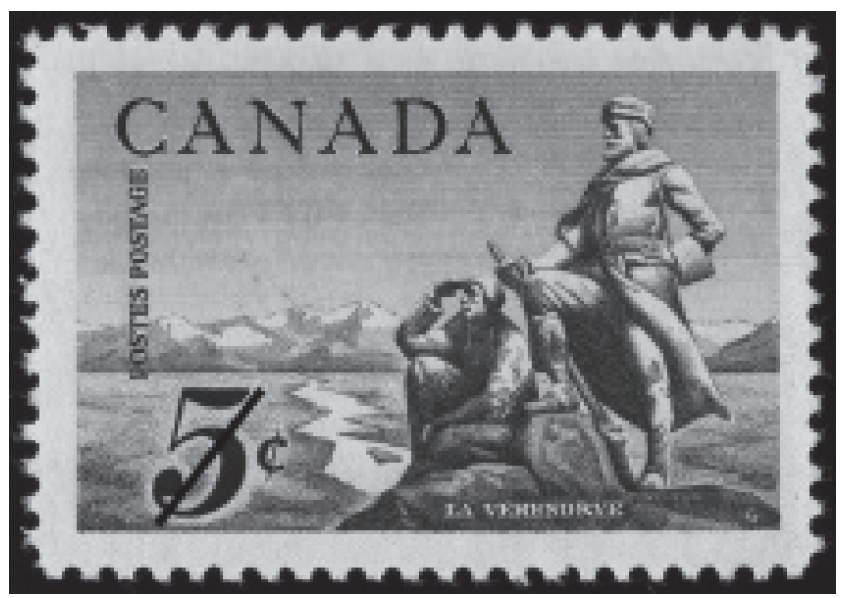

En septembre 1938, un nouveau timbre-poste de 5 cents commémore le bicentenaire de l'arrivée de La Vérendrye au confluent des rivières Rouge et Assiniboine. Des célébrations d'une durée de neuf jours incluent le dévoilement d'un monument, un défilé et un festival tenu au parc Whittier. Le Winnipeg Canoe Club organise une flottille de 200 canots sur la rivière Rouge.

système dans lequel l'esclavage était acceptable, cela ne signifie pas que cet aspect de sa vie devrait être passé sous silence dans la documentation historique, afin que son legs, jusqu'alors idéalisé, puisse demeurer un chapitre agréable du métanarratif du Manitoba, sans jamais être remis en question.

En septembre 1938, un nouveau timbre-poste de 5 cents commémore le bicentenaire de l'arrivée de La Vérendrye au confluent des rivières Rouge et Assiniboine. Des célébrations d'une durée de neuf jours incluent le dévoilement d'un monument, un défilé et un festival tenu au parc Whittier. Le Winnipeg Canoe Club organise une flottille de 200 canots sur la rivière Rouge.

De la même façon, le fait que les concepts de patriarcat et de supériorité blanche auraient prévalu à cette époque ne signifie pas qu'il ne faut pas tenir compte des perceptions de race, des rôles des hommes et des femmes, et des relations entre Autochtones, noirs et Canadiens français. 
À partir de 1731, La Vérendrye et son équipe entrent en contact avec les peuples autochtones de la région, qui font des échanges ou se battent contre eux, cherchant à s'allier en eur faisant la cour ${ }^{21}$. En raison du rôle d'explorateur de La Vérendrye, dépendant des guides autochtones et de leur connaissances géographiques, ses voyages sont un bel exemple de la coopération fragile qui régnait entre les Français, pour la plupart des hommes blancs, et des hommes et femmes autochtones de classes et de statuts sociaux très différents ${ }^{22}$.

Dans une lettre à Maurepas datée du 12 mai 1742, La Vérendryedéclare combienila besoin des guides autochtones «La découverte ne s'est pas faite l'année dernière, faute de guide» ${ }^{23}$. Pour les Français, l'exploration et le commerce dépendaient de la coopération des peuples autochtones. Leur participation était nécessaire pour piéger les animaux et transporter les fourrures, et leur assistance et leur expertise technique étaient essentielles aux explorateurs qui désiraient voyager vers $1^{\prime}$ ouest $^{24}$. Cette coopération avait aussi des implications raciales, que les récits des débuts de la présence française au Manitoba ont tendance à passer sous silence.

À mesure que La Vérendrye et ses compagnons «explorateurs» traversent divers espaces, ils rencontrent de nombreuses nations autochtones très différentes d'eux en termes de vision du monde, de langue et de culture ${ }^{25}$. Des générations antérieures d'historiens ont insisté sur la qualité homogène du Canada français, mais «quand on tient bien compte de tous ceux qui n'étaient ni catholiques, ni français ni blancs, il s'avère que la Nouvelle-France était, dans les faits, une société multiculturelle» [notretraduction] ${ }^{26}$. L'idéologieque partageaient les explorateurs européens, les missionnaires nouvellement arrivés et les rois qui les expédiaient, depuis l'époque de Christophe Colomb jusqu'à la fin decelle de la Nouvelle-France, tenait pour acquis que les «sauvages» autochtones étaient des humains à la culture déficiente qui devraient reconnaitre la valeur présumée et «évidente» de la tutelle européenne ${ }^{27}$. À titre d'exemple, le père jésuite L. F. Nau croyait que les Autochtones étaient incapables de pensée intelligente ${ }^{28}$.

De tels propos racistes étaient monnaie courante. Par exemple, le gouverneur de la Nouvelle-France, Pierre François de Rigaud, marquis de Vaudreuil, s'opposait ouvertement au 
mariage interracial, insistant sur le fait que, «Bad should never be mixed with good... all the Frenchmen who have married savages have been licentious, lazy and intolerably independent; and their children have been characterized by as great a slothfulness as the savages themselves» ${ }^{29}$. Il se peut que La Vérendrye ait été prêt à inclure les Amérindiens dans sa propre catégorie, c'est-à-dire de ceux qui raisonnent et sont humains. Après tout, selon la tradition orale, il a pris pour femme une Anishinabe ${ }^{30}$. Néanmoins, les documents d'archives semblent décrire une autre histoire. En 1738, La Vérendrye dit au marquis de Beauharnois que les Assiniboines, ses «enfants obéissants» présentent leurs hommages à leur "père» ${ }^{31}$.

Cette attitude paternaliste est aussi en évidence quand il dit aux Assiniboins vouloir «to give them intellect,» et quand il informe Beauharnois «I made the same recital to them that I had made to all the others. There was great thankfulness, with many tears and ceremonies, by passing their hands over my head, taking me in your room and place as their father, and our Frenchmen as brothers ${ }^{32}$.» L'idée que La Vérendrye et son équipe d'explorateurs souscrivaient aux idées de supériorité blanche est suggérée par leur empressement d'accepter que le peuple Mandan, qui était censé connaitre l'emplacement de la mer de l'Ouest, était blanc. Quand les deux Français que La Vérendrye avait laissés avec les Mandans pour apprendre leur langue ont été informés par un chef assiniboine qu'une tribu de blancs existait parmi les Mandans, ils ont vite fait de contacter La Vérendrye pour lui dire:

ces gens [...] étaient blancs comme eux qu'ils avaient de la barbe et priaient le grand Maître de la vie dans les livres, en leur dépeignant [... q qu'ils chantaient en tenant leurs livres dans de grandes maisons où ils s'assemblaient pour la prière, et qu'il leur nomma souvent les noms de Jésus et de Marie, en leur montrant une croix qu'il avait au col depuis la naissance ${ }^{33}$.

La Vérendrye et son équipe pouvent facilement s'identifier à cette description d'un supposé groupe blanc, car, toujours selon le chef assiniboine, "leurs villes et forts sont entourés de bonnes murailles avec de grands fossés remplis d'eau, des ponts-levis, portes de fer et beaux remparts ${ }^{34} \gg$. Par surcroit, on leur a raconté que cette tribu blanche utilisait "poudre, canons, fusils, hâches et couteaux,» échangés avec les «sauvages», 
élevait toutes sortes d'animaux, y compris beaucoup de chevaux, travaillait le coton et le fil, et portait des vêtements semblables aux leurs ${ }^{35}$. Il paraissait donc logique pour La Vérendrye que cette tribu «civilisée» de Mandans, qu'on pouvait supposer de culture chrétienne, qui avait des maisons, de la technologie agricole, et qui s'habillait comme eux, aurait la solution pour accèder à la mer de $\mathrm{l}^{\prime}$ Ouest ${ }^{36}$. Ainsi il est très déçu en arrivant à la rivière Missouri, où son groupe se rend compte que le village mandan n'est pas aussi blanc que le leur a laissé croire le chef assiniboine, qui les aurait dupés pour augmenter ses biens et ses profits du fait qu'ils avaient besoin de guides.

Cet exemple illustre parfaitement que les idées de race pouvaient prévaloir en Nouvelle-France, dans certains cas, tutefois, il n'existait pas de limites entre Autochtones, noirs et blancs, tous attachés à leurs terres et à leur communauté partagée, quoique divisés en catégories raciales vagues.

Le concept du «terrain d'entente» de Richard White, une métaphore pour exprimer l'espace social du territoire vaste et changeant situé entre l'Est, de plus en plus habité par des Européens, et l'Ouest, lointain et encore inconnu, peut s'appliquer, quoiqu'avec des réserves importantes, au rapprochement d'individus français et autochtones dans le monde de La Vérendrye ${ }^{37}$. Par un processus d'invention mutuelle, les peuples autochtones et les Canadiens français ont élaboré une série de prémisses qui reflétaient leurs intérêts, y compris la pratique du commerce, qui ont lentement pris les caractéristiques d'une véritable culture. Le 7 aout 1749, le botaniste suédois Peter Kalm (1716-1779) rencontre La Vérendrye et le gouverneur La Galissonnière et dine avec eux à Québec ${ }^{38}$. Kalm est frappé par les éléments de culture autochtone adoptés par la société canadienne-française :

Though many nations imitate the French customs, I observed, on the contrary, that the French in Canada in many respects follow the customs of the Indians, with whom they have constant relations... They follow the Indian way of waging war exactly; they mix the same things with tobacco; they make use of the Indian bark boats... they wrap a square of cloth round their feet, instead of stockings, and have adopted many other Indian fashions ${ }^{39}$. 


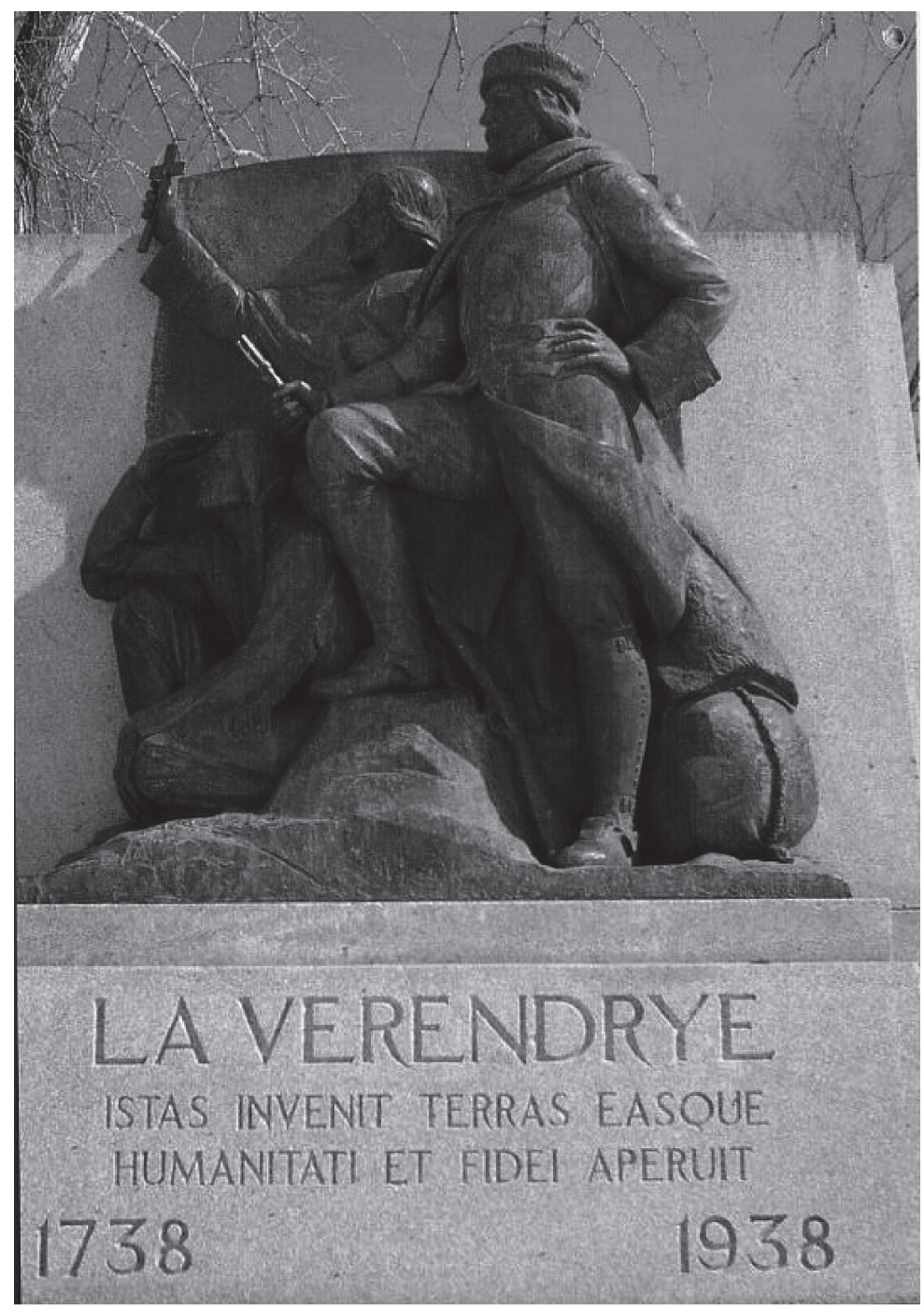

Istas Invenit Terras Easque Humanitati et Fidei Aperuit. Une sculpture de La Vérendrye dévoilée sur l'avenue Taché lors des célébrations du bicentenaire en 1938 portait cette inscription en latin qui signifie «il a découvert ces régions et les a ouvertes à l'humanité et à la foi».

Archives du Manitoba, Sites historiques - La Vérendrye, Saint-Boniface $\mathrm{N}^{\mathrm{o}} 1$. 
La Vérendrye commente sa volonté d'adopter des coutumes autochtones, et il décrit sa frustration devant les Mandans, qui, malgré leur engagement déclaré aux Français, ne rendent aucun service sans être payés en avance et ne voient aucun mal à faire des échanges avec les Anglais, ennemis des Français, dès qu'ils y voient un avantage ${ }^{40}$.

Pendant un certain temps, la tradition autochtone d'échanger des cadeaux servait à forger des alliances avec les Français. ${ }^{41}$ Au début de sa carrière d'explorateur, La Vérendrye raconte une occasion où il reçoit en caeau des cris un esclave et un collier: «In exchange for the slave, I gave a cloak, a shirt, leggings and breeches, a knife and an awl, gun powder and musket balls» ${ }^{42}$. Évidemment, une partie de la tradition de l'échange de cadeaux en Nouvelle-France comprenait des prisonniers de guerre autochtones condamnés à devenir des esclaves dans les maisons canadiennes-françaises ${ }^{43}$.

Les peuples autochtones alliés, en particulier les Outaouais (Odawa) et les Illinois, offraient des prisonniers ennemis de l'ouest aux marchands français associés au commerce des fourrures en guise de symbole puissant de leur partenariat émergeant, et les autorités françaises trouvaient pour leur part que les échanges de prisonniers représentaient un des moyens les plus efficaces de stabiliser les alliances fragiles forgées avec des groupes autochtones. ${ }^{44}$ Futés par rapport à la dimension culturelle de la diplomatie autochtone, qui conférait aux prisonniers un pouvoir symbolique capable de mitiger les effets de la guerre et des meurtres, les Français ont œuvré stratégiquement afin de forger des alliances avec des groupes autochtones qui exprimeraient leur reconnaissance à travers la violence, le commerce des fourrures et des prisonniers qui pourraient être vendus comme esclaves ${ }^{45} \mathrm{Il}$ faut ajouter que les prisonnières avaient un avantage dans les sociétés polygames telles que celle des Illinois, où elles s'intégraient plus facilement que les hommes ${ }^{46}$. De plus, les prisonniers n'étaient pas exclusivement autochtones ou noirs. À titre d'exemple, Antoine Bonnefoy, décrit dans son journal son expérience d'avoir été attaqué et réduit à l'esclavage par les Indiens Cherokee en 17411742. Il explique que quatre Français, dont lui-même, et un homme noir dans son groupe «fumes saisis par chacun un des ces sauvages qui nous fit son esclave. Mis à terre, nous fûmes 
attachés séparément par chacun un collier d'esclave gênés par le col et les bras seulement, sans cependant nous ôter la liberté pour manger et pagayer, lorsqu'il nous a été ordonné de faire dans la suite ${ }^{47}$.» Dans ce cas, des hommes ayant une couleur de peau différente subissent le même sort aux mains des agresseurs cherokee.

Cependant, les notions de race étaient un facteur dans la perception et le traitement des hommes et des femmes au Canada français. Les catégories «homme» et «femme» étaient bien définies dans les sociétés canadienne-française et autochtone, où le travail, la diplomatie, l'organisation sociale et les relations familiales se divisaient selon les sexes. Cela étant dit, il existait de grandes différences parmi les idées concernant le statut des femmes et l'étendue du pouvoir à l'intérieur d'une catégorie sexuelle chez les Canadiens français et les peuples autochtones ${ }^{48}$.

Les Canadiennes françaises et les hommes qui évoquaient la «supériorité» mâle vivaient au début du monde moderne, une époque où on présumait que les femmes, de par leur nature, avaient besoin d'être dominées par les hommes. Tout comme en France, le Canada français était bâti sur une base de patriarcat, où les hommes régnaient sur les femmes, notamment au sein de l'institution de la famille. Pour les femmes, encore plus que pour les hommes, l'état matrimonial était d'importance cruciale. Selon les normes européennes, presque tout le monde sauf le clergé se mariait, et les veufs et veuves se remariaient peu après la mort du premier époux, de sorte que le fait d'être une femme adulte signifiait, dans la grande majorité des cas, être mariée ${ }^{49}$. L'union matrimoniale chez les Canadiens français n'exigeait pas, cependant, que l'identité économique d'une femme soit subsumée sous le nom de son mari. Il arrivait plutôt, comme c'est le cas chez Pierre de La Vérendrye et Marie-Anne du Sablé, qu'un couple forme une "communauté par mariage» de deux personnes, ou «communauté de biens» ${ }^{50}$. La femme anishinabe de La Vérendrye a été gravement humiliée par le mariage de ce dernier à Marie-Anne. Selon la tradition orale, elle aurait tenté d'empoisonner Marie-Anne du Sablé lorsqu'elle accompagna son mari dans $l^{\prime}$ Ouest $^{51}$. Ce mariage, de notoriété publique dans les communautés autochtones du Manitoba, n'a jamais été admis légalement, ni encore reconnu dans la documentation 
historique. Les contrats de mariage sujets au code civil canadienfrançais exigeaient les deux signatures, du mari et de la femme. Mais bien que cela puisse paraitre plutôt égalitaire, la coutume de Paris déclarait sans équivoque que «le mari est le maitre de la communauté» ${ }^{52}$. Quand Marie-Anne du Sablé a signé son contrat de mariage, le notaire a sûrement enregistré qu'elle le faisait avec le consentement de son mari. La communauté de biens conférait donc aux femmes l'égalité devant des droits de propriété, mais non l'égalité des pouvoirs dans le mariage.

Malgré leur subordination envers leur mari, au début

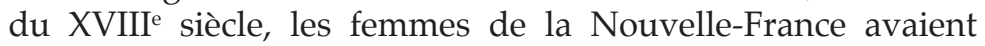
généralement plus de pouvoir et de meilleures opportunités économiques qu'elles en auraient eu en Europe, et ce, en raison de l'absence des hommes attribuable aux guerres, aux efforts d'exploration ou au commerce des fourrures. Aux débuts du Canada français, les femmes blanches avaient un meilleur accès à une éducation supérieure et pouvaient jouer un rôle plus responsable ou participer au commerce. Elles étaient peut-être mieux éduquées que leurs pendants masculins, du fait que la responsabilité maternelle de l'éducation des jeunes enfants aurait pu encourager une plus grande proportion de femmes à développer leurs compétences en lecture ${ }^{53}$.

Il est probable que Marie-Anne ait passé du temps à éduquer les quatre fils et les deux filles qu'elle a eus avec Pierre de La Vérendrye. Les Canadiennes françaises ont aussi apporté une contribution incalculable à l'économie de l'époque, bien qu'il demeure difficile d'évaluer cette contribution. Les documents de l'époque coloniale permettent d'estimer la production de marchandises telles que le blé et les fourrures, manipulées majoritairement par des hommes français, mais les quantités de beurre, de laine ou d'œufs produites par les femmes en Nouvelle-France demeurent un mystère ${ }^{54}$.Le manque de documentation généralisé sur les contributions économiques des femmes reflète le statut secondaire qu'on leur attribuait.

En plus de leurs responsabilités personnelles, les femmes contribuaient souvent de façon directe aux entreprises de leur mari. La Vérendrye faisait confiance aux habiletés de sa femme, qui assumait la responsabilité de ses affaires pendant ses absences. À maintes reprises, il a signé des documents lui accordant le pouvoir de recevoir son salaire ou de gérer les 
fournitures ${ }^{55}$. Selon un expert, avant sa mort en aout 1740, Marie-Anne s'occupait seule des affaires commerciales de La Vérendrye, agissant à titre d'avocat et d'acheteur pendant les voyages de son mari ${ }^{56}$. Souvent La Vérendrye s'en remet à sa femme en tant $\mathrm{qu}^{\prime}$ avocate et procuratrice pendant ses longues absences, ce qui renforce l'affirmation que c'était la famille plutôt que l'individu qui était l'acteur économique primaire pendant les siècles où la France gouvernait le Canada ${ }^{57}$. Pour citer Greer, «La famille formait une équipe, bien qu'avec des membres inégaux $»^{58}$. Chez les femmes, celles qui étaient nobles et religieuses comme Marie-Anne avaient le plus de pouvoir en Nouvelle-France, tandis que les femmes esclaves, dont les deux tiers étaient autochtones, et un tiers noires, étaient les plus soumises. Et malgré cette hiérarchie parmi les femmes, toutes étaient soumises aux hommes.

Dans la correspondance personnelle et officielle de La Vérendrye, plusieurs commentaires sommaires et bribes d'information à la suite de ses rencontres avec ces femmes autochtones, dont bon nombre ne se mariaient pas, donnent un aperçu de l'expérience des femmes et des hommes en Nouvelle -France. La Vérendrye est surpris que les femmes cries aient la responsabilité de porter les biens personnels des hommes, et que les femmes Mandans soient reléguées au même rang que celui des esclaves et des chiens, en portant toutes les fournitures, tandis que, selon ce que voit La Vérendrye, «les hommes ne portent que leurs armes ${ }^{59} \gg$.

Sa notion construite de ce que devrait être le travail des femmes est de nouveau ébranlée quand il raconte son étonnement, lors d'un voyage avec les Assiniboines à la recherche de la nation Mandan: «j'avais tout ce que je voulais porter dans un sac de cuir, que l'une des femmes des guides portait pour moi $^{60}{ }^{»}$. Les commerçants français, les explorateurs et les autorités coloniales s'étonnaient souvent de voir à quel point les femmes autochtones travaillaient fort, et ils présumaient parfois que celles-ci étaient traitées en «bêtes de somme», forcées aux travaux durs par les hommes autochtones ${ }^{61}$. Dans sa description des Mandans, La Vérendrye note que «both men and women of this nation are very laborious ${ }^{62} \gg$. Les Européens auraient dépeint les femmes autochtones comme des «souffredouleurs» pour représenter la sauvagerie amérindienne et ainsi 
aider à justifier la colonisation ${ }^{63}$. En réalité, le stéréotype de la «bête de somme squaw» reflétait le malaise que ressentaient les Canadiens français devant les femmes autochtones dont le travail - fabrication de mocassins, préparation des peaux, contribution à l'alimentation par des poissons et du menu gibier - semblait empiéter sur ce qu'ils croyaient être le domaine des hommes. Les observateurs européens «n'ont pas compris l'étendue des rôles économiques qui revenaient aux femmes, ni le degré auquel elles géraient et dirigeaient leurs propres activités, ni, et $c^{\prime}$ est là le plus important, à quel point elles avaient le droit de possession et de distribution sur ce qu'elles produisaient et transformaient [notre traduction] ${ }^{64}{ }^{\prime}$. Tout comme les hommes et les femmes du Canada français, les hommes et les femmes autochtones avaient des rôles sociaux bien définis. Cependant, ces derniers ne vivaient pas dans un système de patriarcat omniprésent.

Le rôle économique et le statut politique des femmes au sein des communautés autochtones font l'objet de grands débats. Les chercheurs s'entendent pour dire que ce ne sont pas toutes les sociétés autochtones qui étaient égalitaires avant le contact européen, et que leur degré d'égalité pouvait aussi varier $^{65}$. Cependant, ils reconnaissent tous que le contact avec les commerçants européens, les missionnaires et les colons a entrainé ou a intensifié la subjugation des femmes. En effet, dans le cadre de leurs premiers efforts missionnaires au Canada, les Jésuites, notamment le contemporain de La Vérendrye, le père Aulneau, ont fait de leur mieux pour imposer les normes du patriarcat, encourageant les hommes autochtones à battre leurs enfants, à humilier leurs femmes si elles se «révoltaient» et à dominer leurs familles. ${ }^{66}$ Ainsi, «le Canada avait une fonction particulièrement importante dans la pensée des Jésuites au début de l'âge moderne [notre traduction] ${ }^{67}$ ». Pour les Jésuites, le Canada était une terre d'opportunité pour l'épanouissement personnel et celui de la société chrétienne. Une lettre l'explicite «When the French of Canada first entered these fur countries, every summer a priest came to instruct the traders and their men in their religious duties, and preach to them and the [Aboriginals] ${ }^{68}$ ». Le registre de la mission de Michilimakinac mentionne le baptême de deux esclaves, Marie-Madeleine et Joseph, qui appartenaient à la famille La Vérendrye ${ }^{69}$. Mais ces premières tentatives de réorchestrer la société autochtone ont 
connu un succès mitigé, et les missionnaires n'ont pas réussi à réorganiser les normes relatives aux sexes de certaines de ces nations, y compris les Iroquois.

Les normes sexuelles chez les Iroquois contrastent nettement avec celles de la France. Les femmes portaient le fardeau de l'économie familiale, mais en même temps, elles jouissaient du contrôle sur la maisonnée, en particulier sur la nourriture. De plus, puisque la descendance était tracée uniquement à travers la lignée féminine, seules les femmes pouvaient conférer les noms dont les hommes avaient besoin quand on les élevait au statut de chef de clan. Cela leur permettait d'influencer la sélection des chefs ${ }^{70}$. La déclaration du missionnaire jésuite et écrivain Joseph-François Lafitau (1681-1746) sert de témoignage du pouvoir des femmes de la communauté iroquoise de Kahnawaké:

Nothing...is more real than this superiority of women. It is of them that the nation really consists... All real authority is vested in them. The land, the fields, and their harvests all belong to them. They are the souls of the Councils, the arbiters of peace and war. They have charge of the public treasury. To them are given the slaves...The children are their domain, and it is through their blood that the order of succession is transmitted. The men, on the other hand, are entirely isolated. ${ }^{71}$

Bien que le manque de documentation existante sur les femmes autochtones et celles de la Nouvelle-France ait longtemps limité la recherche, cet exemple témoigne du rôle important que jouaient ces femmes dans leurs communautés. Dans les dernières décennies, les voix des femmes autochtones ont été amplifiées par le travail d'historiennes féministes comme Sylvia Van Kirk, dont l'ouvrage Many Tender Ties: Women in Fur-Trade Society, 1670-1870 décrit de quelles façons les femmes autochtones sont devenues essentielles aux Européens en tant que conjointes en raison de leurs compétences techniques et culturelles et de leurs connexions pour le commerce (Combet, 2001).

Le problème demeure que les voix autochtones sont encore subsumées dans des textes qui «reprennent possession» des relations entre les races et des normes sexuelles aux débuts du Canada. Leur rôle n'est que rarement saisi à travers l'histoire orale ou matérielle, il l'est plutôt à travers des documents 
officiels rédigés par des autorités coloniales et les lettres et journaux intimes des commerçants qui ont été retrouvés. Encore plus faibles que les voix des femmes, et même des hommes autochtones, sont les voix des esclaves qui ont peiné en Nouvelle-France. Or il est clair que l'esclavage, la relation de subordination ultime, existait en Nouvelle-France, et l'implication de La Vérendrye dans cette institution doit être admise et examinée.

Les lettres et les rapports de La Vérendrye indiquent qu'il accepte l'esclavage et interagit avec des esclaves de façon régulière. En voyageant avec les Assiniboines à la recherche de la nation Mandan, il écrit, «everything useful for my personal needs was carried by my servant and my slave ${ }^{72} \gg$. En outre, en 1738, il dresse la liste de ceux qui voyagent avec lui: «Notre petite bande était composée de vingt français, M. Lamarque, son frère, deux de mes enfants, mon domestique, un esclave, quatre sauvages avec leurs femmes, nous nous rendîmes aux Mantannes ${ }^{73}$ ». Il écrit également que son cousin, le sieur de La Jemeraye, a acheté trois enfants et les a amenés à Montréal ${ }^{74}$. Tout aussi pertinente est la dépêche du 26 mai 1742 à Beauharnois, dans laquelle le père Claude-Godefroy Coquart rapporte qu'une troupe de guerriers composée de Cris et d'Assiniboines avait récemment écrasé les Sioux des plaines après une bataille de quatre jours, où ils ont tué 70 hommes en plus des femmes et des enfants, et capturé un si grand nombre d'esclaves qu'ils formaient une ligne longue de quatre arpents. ${ }^{75}$ En rapportant cela à Maurepas le 24 septembre 1742, Beauharnois affirme que «this will not be good for La Vérendrye's affairs for he will have more slaves than bundles of fur ${ }^{76}$.» $\grave{A}$ la lumière de ces preuves, Yves Zoltvany et Marcel Trudel, expert reconnu dans le domaine de l'esclavage en Nouvelle-France, ont déclaré catégoriquement que La Vérendrye a amassé et vendu un grand nombre d'esclaves autochtones. Ils citent son mémoire de 1744 à Maurepas, dans lequel il déclare que la colonie a bénéficié de ses activités dans l'Ouest principalement de trois manières: «the great number of people my enterprise provides with a living, he says, the slaves it procures to the colony and the pelts which had previously gone to the English,», y voyant la preuve de ses activités dans le commerce des esclaves ${ }^{77}$. 
Toutefois, l'éminent expert sur La Vérendrye, Antoine Champagne, réfute passionnément cette affirmation, et déclare qu'Yves Zoltvany «ne connaît ici comme preuve que son imagination passionnée... Tout cet alinéa , dit-il, sent l'ignorance du sujet et le fantasme ${ }^{78}$ ». Pourtant, ce démenti par Champagne de la complicité de La Vérendrye dans la traite des esclaves ne résiste pas à un examen critique.

Dans son ouvrage fondamental de 1960, qui examine l'esclavage autochtone et africain au début de la colonisation du Canada, Marcel Trudel argumente que La Vérendrye doit figurer sur la liste de seulement trois hommes qui ont occupé une place prépondérante dans le commerce des esclaves. Trudel critique sévèrement la réaction d'étonnement devant l'idée de l'esclavage au Canada. Dans ses propres mots, il explique:

Dès que nous abordons ce sujet de l'esclavage, on s'écrie: «Comment! De l'esclavage au Canada?». Il faut sans cesse recommencer le même exposé: nous avons eu près de 4000 esclaves, ces esclaves (dont 2 sur 3 étaient des sauvages) ont appartenu à des Canadiens de toutes les classes sociales, on en a fait un commerce pleinement reconnu par les lois... De cette page de notre histoire, le souvenir s'est bien rapidement perdu ${ }^{79}$.

Les documents démontrent que des esclaves ont appartenu à des Canadiens de diverses classes sociales et que le clergé, à qui des esclaves ont été donnés par des acteurs tels que La Vérendrye, était impliqué dans ce commerce. Trudel maintient que La Vérendrye possédait au moins trois esclaves, présente des données qui confirment que ses fils en avaient au moins six, et montre qu'en 1749, Louis-Joseph La Vérendrye a donné un esclave en cadeau à la mission jésuite de Michilimakinac ${ }^{80}$. Bien que le nombre total d'esclaves possédés par La Vérendrye et sa famille paraisse relativement peu élevé, en se basant sur son alliance avec les Assiniboines - «ces grands rabatteurs d'esclaves» - et sur sa déclaration quant au nombre d'esclaves obtenus par les entreprises pour la colonie, on peut déduire qu'il a aidé à alimenter le commerce des esclaves ${ }^{81}$. Par surcroit, la recherche de Trudel suggère que La Vérendrye aurait acheté un esclave noir de la veuve de Philibert en $1748^{82}$. Ainsi, même les femmes possédaient des esclaves. En fait, une parente des La Vérendrye, Marie-Marguerite, fondatrice des Sœurs grises, 
possédait trois des quarante-trois esclaves qui appartenaient au clergé et aux communautés religieuses de la Nouvelle-France ${ }^{83}$.

L'agencéité variait parmi les femmes esclaves autochtones et noires. Si on lit entre les lignes, une des lettres de La Vérendrye met en évidence le fait que les femmes autochtones esclaves trouvaient des ouvertures où elles pouvaient exercer une action malgré leur statut en tant que propriété. La Vérendrye décrit l'attaque du sieur René Bourassa par un groupe de Sioux. Il explique que Bourassa était lié et laissé à la proie du feu quand il a été sauvé par les supplications de son «esclave siouse» qu'il avait toujours bien traitée et qu'il avait sauvée de la mort ${ }^{84}$. Bien que l'explication faite par La Vérendrye de la libération de Bourassa lui donne du sérieux pour sa bonté envers son esclave et fasse de cet incident la raison principale de sa libération, il est clair que l'esclave, qui se trouvait souvent dans une situation qui interdisait l'action par motivation personnelle, a fait le choix de lui épargner une mort pénible.

À Montréal, relativement près de la résidence de La Vérendrye à Trois-Rivières, une femme nommée Marie-Josèphe Angélique, désavantagée par son sexe, sa race et sa position sociale, a résisté à son statut d'esclave noire. Elle a fui ses propriétaires plusieurs fois et c'est peut-être elle qui a mis le feu au Vieux-Montréal le samedi 10 avril 1734, accusation pour laquelle elle a fini par être pendue pour trahison. ${ }^{85}$. Des exemples de l'agencéité de femmes esclaves font pourtant l'exception à la règle. Ces cas demeurent importants, néanmoins, parce qu'ils confirment que ce genre d'acte se produisait, et ils nous offrent un aperçu du niveau de résistance que les esclaves étaient prêtes à manifester pour combattre leur oppression et la discrimination à leur égard. Même si la culpabilité de Marie-Josèphe Angélique en mettant le feu au Vieux-Montréal était largement reconnue comme un fait, un exemple des préjugés raciaux et sexuels du Conseil supérieur de la Nouvelle-France transparait dans la suggestion qu'une simple «négresse» n'aurait pas pu commettre un acte aussi audacieux toute seule ${ }^{86}$. Les relations de l'époque de l'esclavage au début de la colonisation du Canada étaient donc basées sur une brutalité sous-jacente et des démarcations importantes entre les sexes et les races, qui remontent à la surface dans la documentation historique. Une autre forme de brutalité, celle de la guerre dans les années 1730 et 1740, met 
en relief la précarité du «terrain d'entente» dans les relations françaises-algonquines.

De nombreux actes de violence et de meurtres interraciaux ont marqué le paysage de la Nouvelle-France. Deux exemples particulièrement sanglants sont les meurtres du missionnaire jésuite, le père Aulneau, et du fils de La Vérendrye, Jean-Baptiste, par un groupe de Sioux en juin 1736 lorsqu'une troupe de guerriers au lac Des bois a tué vingt-deux membres du groupe de La Vérendrye ${ }^{87}$. Dans son rapport sur cette tragédie, La Vérendrye exprime sa tristesse profonde, et mentionne comment la perte d'un fils a affecté une mère qu'il a vue sur le site de l'évènement: «Il n'y a eu que la calice, qu'une femme avait jeté à la rivière parce que son enfant était mort ${ }^{88}$ ». À l'époque, plusieurs croyaient que les Sioux voulaient se venger du fils de La Vérendrye parce qu'il s'était allié deux ans auparavant aux Cristinaux pour combattre les Sioux. ${ }^{89}$ Ainsi, la vengeance faisait partie intégrante des motivations de groupes indigènes qui cherchaient à exercer des représailles, et La Vérendrye l'a subie directement. Tandis qu'il se liait d'amitié avec des tribus pour des raisons économiques, et en récoltait les bénéfices à travers les guerres entre eux, il était profondément déçu d'avoir échoué à maintenir la paix dans la région. Il écrira, «l'on ne pourra de longtemps pacifier toutes ces nations ayant de mortels ennemis de tout temps ${ }^{90} »$. L'affirmation de Karl Marx selon laquelle les hommes et les femmes font leur propre histoire, mais ils ne la font pas de leur propre mouvement, ni dans des conditions choisies par eux seuls, mais bien dans les conditions qu'ils trouvent directement et qui leur sont données et transmises, s'applique certainement à la Nouvelle-France où existaient la violence, l'esclavage, la discrimination raciale et le patriarcat $^{91}$.

La mort de La Vérendrye en 1749, l'expédition dans le but de «localiser» la mer de l'Ouest a pris fin. Son héritage se fait sentir de bien des façons, plus particulièrement dans son rôle de premier Européen à établir un poste de traite sur le site de ce qui est maintenant la ville de Winnipeg, la capitale grouillante de la province du Manitoba. D’habitude, La Vérendrye est perçu comme un homme de caractère irréprochable et est présenté comme le modèle de tout ce qu'il y avait de mieux au début de la colonisation française du Canada. En 1927, un biographe 
écrira: «Au cours des années depuis la mort de La Vérendrye, il y a eu de nombreuses occasions de vérifier si le moindre degré d'indignité entachait sa nature. Or, rien qui permette de le discréditer n'a été découvert [notre traduction] ${ }^{92}$ ».

Irene Moore, ne tenant pas compte des interactions turbulentes que La Vérendrye a vécues avec les populations autochtones et les tensions qui existaient entre lui et les autorités du gouvernement français au sujet de sa véritable motivation, croit que «les grâces de son esprit et sa personne et l'ampleur de sa performance ont mérité à ce voyageur quasi incomparable l'amour et le souvenir des habitants de sa terre natale et des terres qu'il a découvertes, et ce, jusqu'à la dernière syllabe du temps ${ }^{93} . »$ Sebasant sur ce genre de récit, $l^{\prime}$ histoire conventionnelle a depuis longtemps élevé La Vérendrye en symbole audacieux et héroïque de la première présence française en Amérique du Nord. Cependant, si on veut redonner une sorte d'équilibre à $l^{\prime}$ historiographie relative à cet explorateur du XVIII siècle, il faut examiner son implication dans le commerce des esclaves et le contexte de relations hommes-femmes et de préoccupations raciales dans lequel il exerçait ses activités.

Comme l'a souligné cet article, les composantes non catholiques et non blanches faisaient partie intégrante de la société et de la culture coloniale du Canada français et exerçaient une influence sur elle. Essentiellement, ce sont les hommes qui avaient le pouvoir en Nouvelle-France. En effet, sauf chez les Iroquois et dans certaines enclaves autochtones, où les femmes n'étaient pas soumises à l'autorité des hommes, une forme simple de patriarcat typique des débuts de l'âge moderne prévalait. Cependant, au Canada à cette époque, le principe de base du patriarcat laissait de la place à toutes sortes de complexités, de variantes et de contradictions dans les relations véritables entre hommes et femmes, en particulier quand des hommes comme La Vérendrye s'absentaient pour de longues périodes. Cela étant dit, la Nouvelle-France était loin d'être une société égalitaire. L'esclavage y existait sous une forme institutionnelle tout comme partout ailleurs à travers l'Amérique indienne. Finalement, comme en témoignent les expériences de La Vérendrye, les Canadiens français étaient plongés dans une situation où il était essentiel de coopérer et de négocier avec les Autochtones sur un terrain commun 
toujours changeant et souvent précaire. Les premiers habitants du Canada étaient présents de façon importante et durable en Nouvelle-France, tout comme ils continuent à l'être à l'échelle du Canada de nos jours.

\section{NOTES}

1. Merci aux personnes suivantes pour leur rétroaction judicieuse pendant les premières étapes de ce projet: Adele Perry, Brittany Luby, Carolyn Podruchny et Tom Peace. J'ai mis le terme découverte entre guillemets pour souligner le fait que la terre a été découverte et les routes de commerce établies longtemps avant l'arrivée de La Vérendrye. Les études historiques qui suggèrent le contraire doivent d'être consultées, mais non d'être reproduites. Les termes rencontre et colonisation sont plus exacts que découverte. Je voudrais reconnaitre ici le besoin de déconstruire des termes tels que découverte et explorateur, qui font partie de l'idéologie avancée par les entrepreneurs, les marchands et les proto-capitalistes sous les auspices du clergé, des militaires, et les buts avoués du gouvernement colonial de noble entreprise, buts extrêmement problématiques en soi.

2. Irene Moore dresse une liste longue de deux pages des mémoriaux à La Vérendrye dans Valiant La Vérendrye. Quebec: King's Printer, 1927. On trouve aussi bon nombre de sites, d'hommages et d'images sur le site Web de l'Encyclopédie du patrimoine culturel de l'Amérique française sous «La Vérendrye d'hier à aujourd'hui» et «documents complémentaires».

3. Programme des célébrations du bicentenaire de l'arrivée de Pierre Gaultier de La Vérendrye au confluent des rivières Rouge et Assiniboine, Winnipeg et Saint- Boniface, 3-11 septembre 1938. Winnipeg: S.N., 1938, p. 23, 26. (Texte en anglais seulement.)

4. Dans le contexte de cet article, les mots noir et blanc sont employés comme adjectifs sans égard aux différences entre groupes et identités nationales. Ils ne portent donc pas de majuscule.

5. Voir, par exemple, Allan Greer, The People of New France, dir. Craig Heron et Franca Iacovetta. Toronto: University of Toronto Press, 1998 et Robin Winks, The Blacks in Canada $2^{\text {nd }}$ ed. Montreal: McGill-Queen's University Press, 1997. Les personnes que nous considérons aujourd'hui comme blanches n'étaient pas forcément vues comme telles en Nouvelle-France à cette époque. Il y avait des distinctions importantes entre Grecs, Juifs, etc., sans parler de l'écart majeur entre blancs anglophones et blancs francophones sur le même territoire. 
6. Marcel Trudel, L'esclavage au Canada français: histoire et conditions de l'esclavage. Québec: Presses de l'Université Laval, 1960, p. 331333.

7. Voir Maureen G. Elgersman, Unyielding Spirits: Black Women and Slavery in Early Canada and Jamaica. New York: Garland, 1999, xi, et Brett Rushforth, «A Little Flesh We Offer You: The Origins of Indian Slavery in New France», The William and Mary Quarterly, 2003 vol. $60, \mathrm{n}^{\circ} 4$.

8. Carolyn Podruchny, Making the Voyageur World: Travelers and Traders in the North American Fur Trade. Lincoln: University of Nebraska Press, 2006, p. 7.

9. $\quad$ Ibid., p. 54.

10. Yves F. Zoltvany, «Gaultier de Varennes et de la Vérendrye, Pierre» Dans le Dictionnaire biographique du Canada, vol. III. Sainte-Foy: Presses de l'Université Laval, 1974, p. 266.

11. Ibid., p. 267. Pierre Gaultier de Varennes et de La Vérendrye, Journals and Letters of Pierre Gaultier de Varennes de La Vérendrye and his Sons With Correspondence Between the Governors of Canada and the French Court, Touching the Search for the Western Sea, ed. Lawrence J. Burpee. Toronto: The Champlain Society, 1927, Report of La Vérendrye (1730), p. 50. Voir également Donatien Frémont, Les aborigènes du Nord-Ouest canadien au temps de La Vérendrye. Ottawa: Société Royale du Canada, 1949, p. 15.

12. On trouve un survol plus complet et plus détaillé de la vie et des expéditions de La Vérendrye dans Nellis M. Crouse, La Vérendrye: Fur Trader and Explorer. Toronto: Ryerson Press, 1956; Antoine Champagne, Les La Vérendrye et le poste de l'Ouest. Québec: Les Presses de l'Université Laval, 1968; et Martin Kavanaugh, La Vérendrye: His Life and Times: a Biography and a Social Study of a Folklore Figure, Soldier, Fur Trader, and Explorer. Brandon: Martin Kavanaugh, 1967.

13. Zoltvany, «Gaultier de Varennes et de la Vérendrye, Pierre» pp. 272-273.

14. Voir, par exemple, Archives Nationales de Paris, Col., B, vol. 58, fol.411.

15. Brett Rushforth in «A Little Flesh We Offer You: The Origins of Indian Slavery in New France», The William and Mary Quarterly, Vol. 60, no 4, octobre 2003, p. 53.

16. Crouse, La Vérendrye, p. 98. Voir La Vérendrye, Journals and Letters, «Maurepas à Beauharnois, 22 avril 1737», p. 269-271. Bien qu'on l'ait soupçonné d'être plus préoccupé par le commerce des fourrures et la «mer du Castor» que par l'exploration, La Vérendrye est mort dans la pauvreté. Ses fils ont vendu une partie 
de leurs terrains dans l'Est pour payer les dettes contractées par leur père. Moore, Valiant La Vérendrye, pp. 126-127.

17. Fonds Antoine Champagne 10/56, Société historique de SaintBoniface.

18. Champagne, Les La Vérendrye et le poste de l'Ouest, p. 312.

19. Ibid., p. 358. Bien que les populations créoles des débuts du Canada français soient uniques, leur expérience de l'esclavage est comparable à celle d'autres groupes à travers l'Amérique indienne, un sujet qui mérite un examen plus approfondi. Pendant cette période, toutes les populations humaines étaient petites, et l'esclavage en Nouvelle-France ne doit pas être vu comme un phénomène mineur.

20. Ibid., pp. 358-359.

21. Greer, The People of New France, pp. 120-121.

22. Richard White, The Middle Ground: Indians, Empires, and Republics in the Great Lakes Region, 1650-1815. Cambridge: Cambridge University Press, 1991, p. 51. Comme l'explique White, jusqu'aux années 1730, relativement peu de Françaises se rendaient dans l'Ouest.

23. Fonds Antoine Champagne 10/81/39, Société historique de SaintBoniface.

24. Conrad E. Heidenreich, «French Exploration out of the St. Lawrence Valley», In Germaine Warkentin et Carolyn Podruchny dir. Decentring the Renaissance. Toronto: University of Toronto Press, 2001, p. 249.

25. Podruchny, Making the Voyageur World, p. 302.

26. Greer, The People of New France, p. 76.

27. Ibid.

28. White, The Middle Ground, p. 69.

29. Ibid., p. 70.

30. Brittany Luby, entrevue avec Karlee Sapoznik, 7 mai 2008.

31. Pierre Gaultier de Varennes et de La Vérendrye, North-Western Explorations: Journal of La Vérendrye in the forms of a letter, from the 20th of July, 1738, date of my departure from Michilimakinak, to May, 1739, sent to the Marquis de Beauharnois, Comander of the Military Order of St. Louis, Governor and Lieutenant Governor of whole of New France and country of Louisiana. Canadiana House, 1967, p. 2.

32. Ibid., pp. 3, 5 . 
33. La Vérendrye, Journals and Letters, p. 369.

34. Ibid.

35. Ibid., pp. 369-370.

36. Georges Dugas, The Canadian West: Its Discovery by the Sieur de la Vérendrye; Its Development by the Fur-Trading Companies, Down to the Year 1822. Montréal: Librairie Beauchemin, 1905, pp. 57-58. Dugas propose que «la civilisation» a rendu les Amérindiens plus exigeants. Voir également La Vérendrye, North-Western Explorations, p. 6.

37. Voir White, The Middle Ground, pp. 50-93. Voir également les limites au concept de White d'un terrain commun telles que présentées par Brett Rushforth dans «A Little Flesh We Offer You: The Origins of Indian Slavery in New France», octobre 2003, et l'article de Germaine Warkentin, «Discovering Radisson: A Renaissance Adventurer between Two Worlds», in Reading Beyond Words: Contexts for Native American History, dir. Jennifer S. H. Brown et Elizabeth Vibert. Peterborough: Broadview Press, 1996, p. 48.

38. La Vérendrye, Journals and Letters, p. 27. Voir également Champagne, Les La Vérendrye et le poste de l'Ouest, 296. La Galissonnière écrit une lettre à Maurepas le 23 octobre 1747 pour défendre La Vérendrye. Voir AN, Col., C11E, vol. 16, pp. 300-301.

39. Peter Kalm, Peter Kalm's Travels in North America, trad. Adolph Benson, New York: Dover, 1937. Cité dans Greer, The People of New France, p. 84.

40. Burpee, Journals and Letters, «Report of La Vérendrye 31 octobre 1744, pp. 454-455, et Frémont, Les aborigènes du Nord-Ouest canadien au temps de La Vérendrye, pp. 15, 18.

41. Rushforth, «A Little Flesh We Offer You», p. 59.

42. Denis Combet, À la recherche de la mer de l'Ouest: Mémoires choisis de La Vérendrye. Winnipeg: Great Plains Publications, 2001, p. 57.

43. Brett Rushforth, « lavery, the Fox Wars, and the Limits of Alliance», The William and Mary Quarterly, 2006, vol. 63, n 1, p. 8.

44. Ibid., 27. En provoquant l'épuisement des ressources, le commerce des fourrures a intensifié les rivalités et les guerres entre groupes autochtones. Voir André Champagne, L'histoire du régime français, (Québec: Éditions du Septentrion et Société Radio-Canada, 1996, p. 90.

45. Ibid., pp. 14,34 .

46. Rushforth, «A Little Flesh We Offer You», p. 12. 
47. Antoine Bonnefoy, Fonds Antoine Champagne 10/87/1-2, Société historique de Saint-Boniface.

48. Podruchny, Making the Voyageur World, p. 249.

49. Greer, The People of New France, p. 64.

50. Ibid., p. 69.

51. Luby, Entrevue, le 7 mai 2008.

52. Greer, The People of New France, p. 70.

53. Ibid., p. 67.

54. bid.

55. Voir par exemple, Fonds Antoine Champagne, 10/79/24 et $10 / 80 / 22$ «Procuration de M. de La Vérendrye à son épouse, 11 juin 1735» ainsi que 10/79/30-31, Société historique de Saint-Boniface.

56. Combet, À la recherche de la mer de l'Ouest, p. 117.

57. Greer, The People of New France, p. 68.

58. Ibid.

59. Combet, À la recherche de la mer de l'Ouest, 63 and 105. Voir également La Vérendrye, North-Western Explorations, p. 6.

60. Ibid., p. 103.

61. Podruchny, Making the Voyageur World, p. 251.

62. La Vérendrye, North-Western Explorations, p. 10.

63. Podruchny, Making the Voyageur World, p. 251.

64. Ibid. Produchny reprend cette idée de Priscilla Buffalohead.

65. Ibid., p. 250.

66. Greer, The People of New France, p. 63.

67. Peter A. Goddard, "Canada in Seventeenth-Century Jesuit Thought», in Decentring the Renaissance, dir. Germaine Warkentin et Carolyn Podruchny. Toronto: University of Toronto Press, 2001, pp. 197-198.

68. Voir David Thompson, Fonds Antoine Champagne 10/40, Société historique de Saint-Boniface.

69. Mgr. Cyprien Tanguay, Dictionnaire généalogique des familles canadiennes (Montréal, 1887), III, p. 606.

70. Greer, The People of New France, p. 62. 
71. Joseph F. Lafitau, Mœurs des sauvages américains comparées aux mours des premiers temps. Paris, 1724. Cité dans The People of New France, p. 62.

72. Sylvia Van Kirk, Many Tender Ties: Women in Fur Trade Society, 16701870. Winnipeg: Watson and Dwyer Publishing, 1980.

73. La Vérendrye, North-Western Explorations, 5. Voir également Fonds Antoine Champagne 10/87/30, Société historique de SaintBoniface.

74. Ibid., $10 / 79 / 42-43$.

75. Zoltvany, «Gaultier de Varennes et de la Vérendrye, Pierre», 6. Un arpent équivaut approximativement à un acre, ou le tiers d'un hectare. Voir La Vérendrye, Journals and Letters, «Beauharnois to Maurepas, 24 septembre 1742», pp. 380-382.

76. Ibid. Voir également Combet, À la recherche de la mer de l'Ouest, p. 129.

77. Voir Marcel Trudel, L'esclavageau Canada français: histoireet conditions de l'esclavage. Québec: Presses de l'Université Laval, 1960, pp. 72, 333. Zoltvany, "Gaultier de Varennes et de la Vérendrye, Pierre», 8; La Vérendrye, Journals and Letters, «Report of La Vérendrye» 31 octobre 1744 , pp. 451-452.

78. Fonds Antoine Champagne 10/43, Société historique de SaintBoniface.

79. Trudel, L'esclavage au Canada français, p. 333.

80. Ibid., p. 145.

81. Ibid.

82. Ibid., p.121.

83. Ibid., pp. 154-156.

84. Champagne, Les La Vérendrye et le poste de l'Ouest, 181. Voir La Vérendrye, Journals and Letters, «Beauharnois to Maurepas, 14 octobre 1736», p. 212.

85. Voire Afua Cooper, The Hanging of Angélique: The Untold Story of Canadian Slavery and the Burning of Old Montreal. Toronto: Harper Collins Ltd., 2006.

86. Ibid., p. 88.

87. Crouse, La Vérendrye, pp. 107-108. Voir La Vérendrye, Journals and Letters, " ffair of the murder of twenty-one voyageurs at the Lake of the Woods, in the month of June 1736», pp. 262-266. 
88. Fonds Antoine Champagne 10/89/14, Société historique de SaintBoniface.

89. Ibid., $10 / 80 / 30$.

90. Ibid., $10 / 97 / 32$.

91. Karl Marx, Eighteenth Brumaire of Louis Bonaparte. (Le 18 Brumaire de Louis Bonaparte) New York, 1963, p. 15.

92. Moore, Valiant La Vérendrye, p. 13.

93. Ibid., p. 131.

\section{BIBLIOGRAPHIE}

COMBET, Denis (2001) À la recherche de la mer de l'Ouest: Mémoires choisis de La Vérendrye, Winnipeg, Great Plains Publications.

HEIDENREICH, Conrad E. (2001) «French Exploration out of the St. Lawrence Valley», dans WARKENTIN, Germaine et PODRUCHNY, Carolyn ( dir.) Decentring the Renaissance, Toronto, University of Toronto Press, p. 249-

PODRUCHNY, Carolyn, (2006) Making the Voyageur World: Travelers and Traders in the North American Fur Trade, Lincoln, University of Nebraska Press.

RUSHFORTH, Brett (2003) «A Little Flesh We Offer You: The Origins of Indian Slavery, New France», The William and Mary Quarterly, 2003 vol. $60, \mathrm{n}^{\circ}$, p $4-53$

TRUDEL, Marcel (1960) «L'esclavage au Canada français», dans Histoire et conditions de l'esclavage, Québec:, Presses de l'Université Laval, p. 331-333.WINKS, Robin (1997) The Blacks in Canada $2^{\text {nd }} \mathrm{ed}$. Montreal: McGill-Queen's University Press

WINKS, Robin (1997) The Blacks in Canada $2^{\text {nd }}$ ed. Montreal: McGillQueen's University Press 